\section{Clarifying the relationship between metformin, acute kidney injury and lactic acidosis}

\author{
Samira Bell, Enrique Soto-Pedre, Paul Connelly, Shona Livingstone \\ and Ewan Pearson
}

We read, with interest, the News \& Views article by C. Rhee and K. Kalantar-Zadeh (Diabetes mellitus: Complex interplay between metformin, AKI and lactic acidosis. Nat. Rev. Nephrol. 13, 521-522 (2017)) $)^{1}$, which discusses our recent work looking at the relationship between metformin, acute kidney injury (AKI) and lactic acidosis ${ }^{2,3}$. In response to this discussion of some of our findings, we would like to highlight several points.

First, Connelly et al. demonstrated that $80 \%$ of lactic acidosis within the study cohort occurred in the presence of $\mathrm{AKI}^{3}$. In view of the effectiveness of treatment and increasing evidence of the beneficial cardiovascular effects of metformin, we feel that metformin should be only temporarily discontinued in patients with a condition that predisposes them to acute AKI; this advice is similar to the advice given for angiotensin-converting enzyme (ACE) inhibitors and angiotensin receptor blockers.

Second, although we acknowledge the difficulties of accurately identifying metforminassociated lactic acidosis (MALA) cases in observational studies, we do not agree that the crude incidence rate of lactic acidosis observed in the study by Connelly et al. is biased because it differs from rates reported by others. Instead, we would like to emphasize that crude incidence rates disregard the structure of the population. Thus, comparing crude incidence rates alone can be misleading before standardization is carried out to remove the effect of differential structures in populations under comparison.

Third, it was also suggested that the absence of a 'new-user' design in the study by Bell et al. ${ }^{2}$ may not have accounted for patients who stopped using metformin or died due to its adverse effects before study entry, biasing results towards a protective effect. Our study population comprised $63 \%$ incident users over a long period, making survivor bias a less likely explanation of our findings.

Fourth, the elevated risk of developing AKI that is associated with 'ever' having been on or currently being on metformin is due to within and between-person allocation bias, where being prescribed metformin is a marker for other comorbidities that make the patient more vulnerable to AKI. The fact that AKI incidence is not elevated in patients during periods on the drug versus periods off the drug suggests that the drug itself is not increasing the risk of AKI.
Finally, we do not agree with the recommendation made by C. Rhee and K. KalantarZadeh that the use of metformin should be restricted in patients with chronic kidney disease (CKD), particularly in view of the increasing evidence base that supports metformin safety in patients with $\mathrm{CKD}^{4,5}$. We eagerly await further pharmacokinetic studies on the safety and efficacy of metformin in these patients.

Samira Bell is at the Renal Unit, Ninewells Hospital, Dundee DD 1 9SY, UK; and at Population Health Sciences, School of Medicine, The Mackenzie Building, Kirsty Semple Way, Dundee DD2 4BF, UK.

Enrique Soto-Pedre, Paul Connelly and Ewan Pearson are at the Division of Molecular \& Clinical Medicine, Level 5, Mailbox 12, Ninewells Hospital and Medical School, Dundee DD1 9SY, UK.

Shona Livingstone is at Population Health Sciences, School of Medicine, The Mackenzie Building, Kirsty Semple Way, Dundee DD2 4BF, UK.

Correspondence to S.B. samira.bell@nhs.net

doi:10.1038/nrneph.2017.172 Published online 13 Dec 2017

1. Rhee, C. M. \& Kalantar-Zadeh, K. Diabetes mellitus: complex interplay between metformin, AKI and lactic acidosis. Nat. Rev. Nephrol. 13, 521-522 (2017).

2. Bell, S. et al. Risk of acute kidney injury and survival in patients treated with metformin: an observational cohort study. BMC Nephrol. 18, 163 (2017).

3. Connelly, P. J. et al. Acute kidney injury, plasma lactate concentrations and lactic acidosis in metformin users: a GoDarts study. Diabetes Obes. Metab. 19, 1579-1586 (2017).

4. Ekstrom, N. et al. Effectiveness and safety of metformin in 51675 patients with type 2 diabetes and different levels of renal function: a cohort study from the Swedish National Diabetes Register. BMJ Open 2, e001076 (2012).

5. Roumie, C. L. et al. Comparative effectiveness of sulfonylurea and metformin monotherapy on cardiovascular events in type 2 diabetes mellitus: a cohort study. Ann. Intern. Med. 157, 601-610 (2012).

Competing interests statement The authors declare no competing interests. 